\title{
Diagrams of stability of circumbinary planetary systems
}

\author{
Elena Popova \\ Pulkovo Observatory of the Russian Academy of Sciences \\ Pulkovskoye ave. 65, Saint Petersburg 196140, Russia \\ email: m02pea@gmail.com
}

\begin{abstract}
The stability diagrams in the "pericentric distance - eccentricity" plane of initial data are built and analyzed for Kepler-38, Kepler-47, and Kepler-64 (PH1). This completes a survey of stability of the known up to now circumbinary planetary systems, initiated by Popova \& Shevchenko (2013), where the analysis was performed for Kepler-16, 34, and 35. In the diagrams, the planets appear to be "embedded" in the fractal chaos border; however, I make an attempt to measure the "distance" to the chaos border in a physically consistent way. The obtained distances are compared to those given by the widely used numerical-experimental criterion by Holman \& Wiegert (1999), who employed smooth polynomial approximations to describe the border. I identify the resonance cells, hosting the planets.
\end{abstract}

Keywords. Celestial mechanics, planetary systems, methods: numerical.

The systems Kepler-16, 34, and 35 were considered by Popova \& Shevchenko (2013): stability diagrams were constructed in the plane of initial parameters "pericentric distance - eccentricity", which showed that all three planets are situated in resonance cells bounded by unstable resonances. Here we consider the planetary dynamics in the systems Kepler-38, 47, and 64. The parameters of the systems were determined by Orosz et al. (2012a), Orosz et al. (2012b), Kostov et al. (2013).

To explore the stability problem for the planetary motion, we use two stability criteria, following Popova \& Shevchenko (2013). The first one is the "escape-collision" criterion and the second one is based on the value of the maximum Lyapunov characteristic exponent (maximum LCE). The computations are performed using the algorithms and codes by von Bremen et al. (1997), Shevchenko \& Kouprianov (2002), Kouprianov \& Shevchenko (2003), Kouprianov \& Shevchenko (2005). The employed statistical method for separation of regular and chaotic orbits was proposed by Melnikov \& Shevchenko (1998), Shevchenko \& Melnikov (2003).

Holman \& Wiegert (1999) obtained an empirical formula for the critical semimajor axis (separating the chaotic and regular domains) in function of the binary mass ratio and the binary eccentricity, for zero eccentricity planetary orbits. In Fig. 1(d) the HolmanWiegert $a_{\mathrm{cr}}$ values are given in column 2 , whereas our numerical estimates $a_{\mathrm{cr}}^{\prime}$ (the main border at zero eccentricity) in column 3 .

The computed stability diagrams for Kepler-38, 47, and 64 are given in Fig. 1. The chaotic domains revealed by the maximum LCE criterion are shown in black, and those revealed by the "escape-collision" criterion are shown in gray. Triangles are the nominal planet positions.

Our basic conclusions are as following. ( $i$ ) The planets turn out to be situated in resonance cells between the "teeth" of instability corresponding to the resonances between the orbital periods of the planet and binary (5/1 and 6/1 for Kepler-38b, $6 / 1$ and $7 / 1$ for 


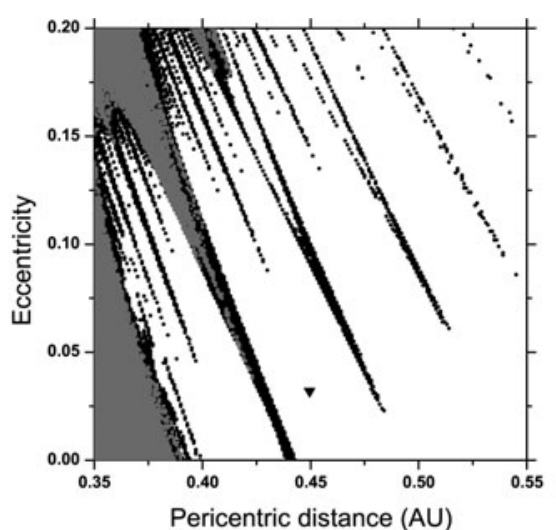

a)

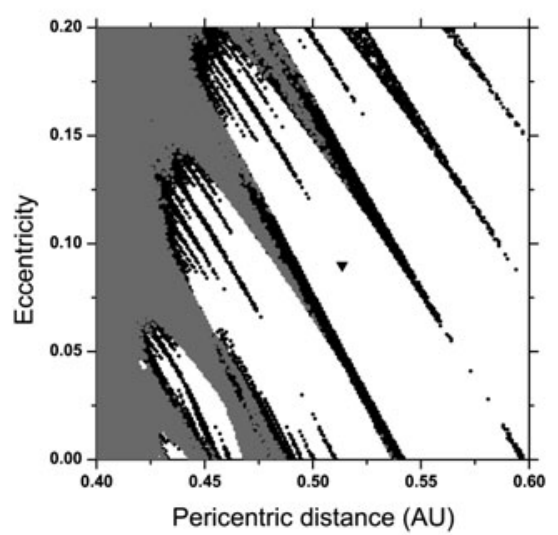

c)

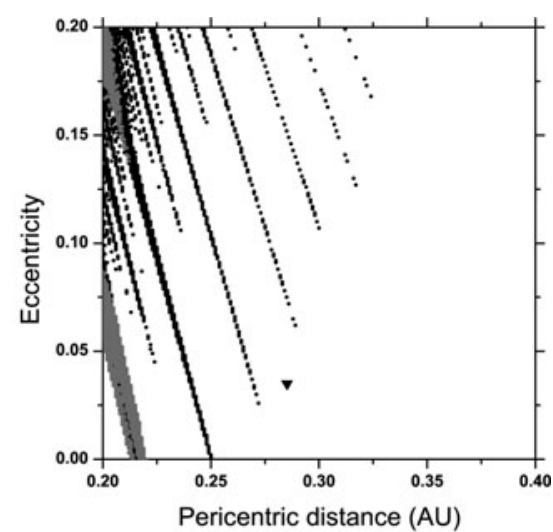

b)

\begin{tabular}{|c|c|c|}
\hline & \multicolumn{2}{|c|}{$\left|a_{\mathrm{cr}}, \mathrm{AU}\right| a_{\mathrm{cr}}^{\prime}, \mathrm{AU}$} \\
\hline Kepler-16b & $\mid 0.646$ & 0.619 \\
\hline Kepler-34b & $\mid$\begin{tabular}{|l}
0.836 \\
\end{tabular} & 0.755 \\
\hline Kepler-35b & \begin{tabular}{|l}
$\mid$ \\
0.497
\end{tabular} & 0.481 \\
\hline Kepler-38b & \begin{tabular}{|l|l|}
0.389 \\
\end{tabular} & 0.395 \\
\hline Kepler-47b & \begin{tabular}{|l|}
0.202 \\
0.2
\end{tabular} & 0.178 \\
\hline Kepler-64b & 0.495 & 0.434 \\
\hline
\end{tabular}

d)

Figure 1. The stability diagrams for (a) Kepler-38, (b) 47, and (c) 64. (d) The critical semimajor axis values from Holman-Wiegert $\left(a_{c r}\right)$ and from stability diagrams $\left(a_{c r}^{\prime}\right)$.

Kepler-47b and 64b). Thus all the circumbinary planets are "embedded" in the fractal chaos border in the stability diagrams. (ii) The semimajor axis critical values, given by the Holman-Wiegert empirical criterion, may differ significantly from real ones (directly obtained from the stability diagrams). (iii) The measured distances between the planet locations and the nearest unstable resonant "teeth" do not exceed $6 \%$ of the planet semimajor axes. (iv) The representative values of the Lyapunov time in the chaotic domains for the studied planets are very small: $1-1.5$ years.

I am grateful to Ivan Shevchenko for useful remarks and advices. This work was supported in part by the Russian Foundation for Basic Research (project No. 14-02-00464).

\section{References}

Popova, E. \& Shevchenko, I. 2013, ApJ, 769, 152

Holman, M. \& Wiegert, P. 1999, AJ, 117, 621

Orosz, J., Welsh, W., Carter, J. et al. 2012, ApJ, 758, 82

Orosz, J., Welsh, W., Carter, J. et al. 2012, Science, 337, 1511

Kostov, V., McCullough, P., Hinse, T. et al. 2013, ApJ, 770, 52

von Bremen, H., Udwadia, F., \& Proskurowski, W. 1997, Physica D, 101, 1 
Shevchenko, I. \& Kouprianov, V. 2002, A\& $A$ A, 394, 663

Kouprianov, V. \& Shevchenko, I. 2003, A\&AA, 410, 749

Kouprianov, V. \& Shevchenko, I. 2005, Icarus, 176, 224

Melnikov, A. \& Shevchenko, I. 1998, Sol. Sys. Res., 32, 480

Melnikov, A. \& Shevchenko, I. 2003, JETP Lett., 77, 642 